\title{
Newborn Respiratory Rate
}

National Cancer Institute

\section{Source}

National Cancer Institute. Newborn Respiratory Rate. NCI Thesaurus. Code C87104.

The number of inhalations and exhalations of the newborn per minute; the normal range is 30 - 60 breaths per minute. 